water and $60 \%$ alcohol ( $5 \mathrm{ml} / \mathrm{kg}$ once per day) by intragastric administration in the first week; $10 \%$ alcohol ad libitum as the drinking water and $60 \%$ alcohol $(10 \mathrm{ml} / \mathrm{kg}$ twice per day) by intragastric administration in the second week; $20 \%$ alcohol ad libitum as the drinking water and $60 \%$ alcohol $(15 \mathrm{ml} / \mathrm{kg}$ twice per day) by intragastric administration from week 3 to week 16; and $30 \%$ alcohol ad libitum as the drinking water and $60 \%$ alcohol $(15 \mathrm{ml} / \mathrm{kg}$ twice per day) by intragastric administration from week 17 to month 6. Animals in the control group received purified drinking water in the same regimen with alcohol treatment. Before and 6 months after initiating the study, left ventricular end diastolic diameter (LVEDD), left ventricular ejection fraction (LVEF), and fractional shortening (FS) were assessed by echocardiography. Six months after the study started, histopathology and ultrastructure of myocardium were examined with light and electron microscopy; mRNA expression of TACE was evaluated by real-time PCR; and protein expression of TACE and TNF- $\alpha$ was analysed using immunohistochemistry and western blot, respectively.

Results Following 6 months of alcohol feeding, LVEF and FS were reduced ( $p<0.05$ for all), while LVEDD was augmented in the ACM group $(p<0.05)$, as compared with the control group. Severe changes in cardiac structure were also seen in the ACM group. The mRNA and protein expression of TACE and the protein expression of TNF$\alpha$ were up-regulated in the ACM group in comparison with the control group ( $p<0.05$ for all). In both groups, the protein expression of TACE positively correlated with that of TNF- $\alpha(p<0.01)$ and LVEDD, whereas it negatively correlated with LVEF $(p<0.05)$.

Conclusions TACE is over-expressed in the ventricle of ACM rats, and may involve in the process of ventricular remodelling via cleaving TNF- $\alpha$. Therefore, TACE may represent a new therapeutic target in the prevention and treatment of ventricular remodelling in ACM.

\section{e0155 SMOKING TREATMENT INCREASE SERUM AGES LEVEL AND HAVE EFFECTS ON EXPRESSION OF ICAM-1 IN VASCULAR ENDOTHELIAL CELLS OF RAT}

doi:10.1136/hrt.2010.208967.155

Yungen Jiao, Naifen Liu. Cardiovascular Institute of Southeast University, Nanjing, China

Objective To investigate whether smoking can increase serum advanced glycosylation end products (AGEs) level and have effects on expression of intercellular cell adhesion molecule-1 (ICAM-1) in vascular endothelial cells of rat.

Methods Male SD rats $(n=138)$ were randomly assigned to five groups according to duration of smoking treatment: 2-week group, 4-week group, 6-week group, 8-week group, smoking cessation group. The rats of following groups, that is $2 \mathrm{w}, 4 \mathrm{w}, 6 \mathrm{w}$ and $8 \mathrm{w}$ groups were further randomly divided into five subgroups according to intervening condition: control subgroup, smoking treatment for $1 \mathrm{~h} /$ per day subgroup, smoking treatment for $0.5 \mathrm{~h} /$ per day subgroup, aminoguanidine hydrochloride subgroup, puerarin subgroup; the rats of smoking cessation group were further randomly divided into three subgroups according to duration of smoking cessation: smoking cessation 2 weeks subgroup, smoking cessation 4 weeks subgroup and smoking cessation 6 weeks subgroup. The rats of $2 \mathrm{w}, 4 \mathrm{w}, 6 \mathrm{w}$ and $8 \mathrm{w}$ groups were sacrificed after smoking treatment for 2, 4, 6 and 8 weeks respectively; the rats of smoking cessation group were sacrificed after smoking treatment for 8 weeks and then cease-smoking for 2,4 , and 6 weeks respectively. Serum AGEs levels of each rat were assayed by fluorescent method. ICAM-1 mRNA and protein of vascular endothelial cells were determined by semiquantitative RT-PCR (Reverse transcription PCR) and immunohistochemistry.
Results Serum AGEs levels of all SM1 subgroups rats were increased after smoking treatment for 2 weeks $(p<0.01)$, and reached peak at 4 weeks $(p<0.001)$, then declined at 6 weeks and 8 weeks, but did not recovere back to normal level; the increasing trend was depressed by aminoguanidine hydrochloride and puerarin. Levels of serum AGEs declined in smoking cessation rats, and were significantly lower at 4 weeks than those before smoking cessation $(p<0.001)$. With the increased duration of smoking, ICAM-1 mRNA and protein of vascular endothelial cells were up-regulated, both aminoguanidine hydrochloride and puerarin depress the up-regulation. The expression of ICAM-1 mRNA and protein of vascular endothelial cells also declined after smoking cessation, and they were significantly lower in rats of smoking cessation of 4 weeks subgroup than those before smoking cessation $(p<0.05)$.

Conclusions Smoking treatment increase serum AGEs level in rat. Cigarette-induced AGEs play roles in the augmented expression of ICAM-1 in vascular endothelial cells of rat with smoking treatment. Aminoguanidine hydrochloride, puerarin and smoking cessation contribute to the decrease of serum AGEs level and the expression of ICAM-1 in vascular endothelial cells of rat.

\section{e0156 UROTENSIN II PROMOTES MONOCYTE CHEMOATTRACTANT PROTEIN-1 EXPRESSION IN AORTIC ADVENTITIAL FIBROBLASTS OF RAT}

doi:10.1136/hrt.2010.208967.156

${ }^{1}$ Zhang Yonggang, ${ }^{1}$ Bao Shilin, ${ }^{1}$ Kuang Zejian, ${ }^{1}$ Ma Yanjun, ${ }^{2}$ Wei Ruihong, ${ }^{1}$ Wu Libiao, ${ }^{1} \mathrm{Hu}$ Yanchao, ${ }^{1}$ Mao Yanyan. ${ }^{1}$ First Affiliated Hospital, Shantou University Medical College, Shantou, China; ${ }^{2}$ Second Affiliated Hospital, Shantou University Medical College, Shantou, China

Background Recent studies reported that vascular adventitial fibroblasts (AFs) are involved in the development of vascular inflammatory diseases, such as atherosclerosis. Urotensin II (UII), a potent vasoconstrictive peptide, could stimulate phenotype differentiation and proliferation of the AFs. The goal of this study was to investigate the effect of UII on the expression of monocyte chemoattractant protein-1 (MCP-1) in rat aortic AFs, and to study the signal transduction pathways of it.

Methods Growth-arrested AFs were incubated in serum-free medium with UII $\left(10^{-10}-10^{-7} \mathrm{~mol} / \mathrm{l}\right)$. In order to explore the mechanism of UII effect, the cells were pretreated with some inhibitors of signal transduction pathways for $30 \mathrm{~m}$, and then incubated with UII $\left(10^{-8} \mathrm{~mol} / \mathrm{l}\right)$ for $3 \mathrm{~h}$ to $24 \mathrm{~h}$. The MCP- $1 \mathrm{mRNA}$ and protein expression induced by UII were evaluated by the reverse transcriptase PCR and Western Blotting, respectively. The MCP-1 secretion from the cells was determined by ELISA.

Results UII could upregulate MCP-1 expression significantly. The MCP-1 mRNA expression increased after $1 \mathrm{~h} \quad(\mathrm{p}<0.05)$ of UII $\left(10^{-8} \mathrm{~mol} / \mathrm{l}\right)$ treatment and reached a peak at $3 \mathrm{~h}(\mathrm{p}<0.01)$. It then declined from 6 to $24 \mathrm{~h}$, and there are no significantly differences from $0 \mathrm{~h}$ group. UII dose-dependently induced MCP-1 mRNA expression, with maximal effect at a concentration of $10^{-8} \mathrm{~mol} / \mathrm{l}$ at $3 \mathrm{~h} \quad(\mathrm{p}<0.01)$. The MCP-1 mRNA expression was increased by $70.10 \%, 109.65 \%, 189.73 \%$ and $122.99 \%$ in $10^{-10} \mathrm{~mol} / \mathrm{l}, 10^{-9} \mathrm{~mol} / \mathrm{l}$, $10^{-8} \mathrm{~mol} / \mathrm{l}$ and $10^{-7} \mathrm{~mol} / 1$ group, respectively, as compared with the control group (without UII stimulation), and the upregulation was significant $(p<0.01$ in all groups). The effect of UII was inhibited significantly by the UII receptor antagonist SB710411 $\left(10^{-6} \mathrm{~mol} / \mathrm{l}\right)$, Rho protein kinase inhibitor Y27632 $\left(10^{-5} \mathrm{~mol} / \mathrm{l}\right)$, protein kinase C inhibitor $\mathrm{H} 7\left(10^{-5} \mathrm{~mol} / \mathrm{l}\right)$, mitogen-activated protein kinase inhibitor PD98059 $\left(10^{-5} \mathrm{~mol} / \mathrm{l}\right)$, calcineurin inhibitor Cyclosporine A $\left(10^{-5} \mathrm{~mol} / \mathrm{l}\right)$ and $\mathrm{Ca}^{2+}$ channel blocker nicardipine $\left(10^{-5} \mathrm{~mol} / \mathrm{l}\right)$, $(\mathrm{p}<0.01$ in all groups). In addition, UII also induced protein expression and secretion of MCP-1 in the cells, both in 
a dose-dependent and time-dependent manner, with maximal effect at a concentration of $10^{-8} \mathrm{~mol} / \mathrm{l}$ at $12 \mathrm{~h}$ (in the level of protein secretion from the cells, $\mathrm{p}<0.01$ ) or $24 \mathrm{~h}$ (in the level of protein expression in the cells, $\mathrm{p}<0.01$ ), which could also be inhibited by these inhibitors ( $p<0.01$ in all groups).

Conclusion Urotensin II may stimulate the expression of monocyte chemoattractant protein-1 in rat aortic adventitial fibroblasts, through its receptor and the $\mathrm{Ca}^{2+}$ channel, protein kinase $\mathrm{C}$, mitogen-activated protein kinase, calcineurin and Rho kinase signal transduction pathways, contributing to the vascular inflammation.

\section{e0157 RNA INTERFERENCE TARGETING ACE AND AT1R GENE REDUCED BLOOD PRESSURE AND IMPROVED MYOCARDIAL REMODELLING IN SHR}

doi:10.1136/hrt.2010.208967.157

Zhou Hua, Gao Fen, Bian Yunfei, Li Maolian, Oiu Ling, Wu Weidong, He Junhua, Xiao Chuanshi. The Second Hospital of Shanxi Medical University, Taiyuan, Shanxi, China

Introduction Angiotensin-converting enzyme (ACE) and angiotensin II (Ang II) Type 1 receptor (ATIR) have been shown to play an important role in the pathogenesis of hypertension.

Objective To investigate the effects of RNA interference (RNAi) ATIR and ACE on blood pressure and myocardial hypertrophy in spontaneously hypertensive rats (SHR).

Methods SHRs were treated with normal saline as vehicle controls, with Ad5-EGFP as vector controls, with recombinant adenoviral vectors Ad5-EGFP-ACE-shRNA carrying shRNA for ACE as ACERNAi, Ad5-EGFP-AT1R-shRNA carrying shRNA for AT1R as AT1RRNAi, and Ad5-EGFP-ACE-AT1R-shRNA carrying shRNA for ACE and AT1R as ACE-AT1R-RNAi. WKY rats were taken as normotensive controls treated with normal saline. Systolic blood pressure of the caudal artery was recorded. Serum levels of ACE and Ang II were determined with ELISA. ACE and AT1R mRNA and protein level were determined in myocardium, aorta, kidney and lung. On day-40 of the experiment, heart was pathologically and ultrastructurly examined. The ratio of heart weight to and left ventricular weight to body weight were calculated.

Results Serum concentration of ACE was lower in ACE-RNAi rats, AT1R-RNAi rats and ACE-AT1R-RNAi rats respectively than in vehicle and vector controls (both $\mathrm{p}<0.05$ ). Serum concentration of Ang II was significantly lower in ACE-RNAi rats and higher in AT1R-RNAi rats than in vehicle and vector controls $(p<0.05)$. The expressions of ACE and AT1R mRNA and protein were significantly reduced in the myocardium, aorta, kidney and lung in ACE-RNAi rats, AT1R-RNAi rats and ACE-AT1R-RNAi rats respectively, compared with that in vehicle and vector controls (all $p<0.05$ ). ACE-RNAi, AT1R-RNAi and ACE-AT1R-RNAi treatments resulted in a reduction of systolic blood pressure by $(22 \pm 6) \mathrm{mm} \mathrm{Hg}$, $(20 \pm 5) \mathrm{mm} \mathrm{Hg},(23 \pm 7) \mathrm{mm} \mathrm{Hg}$ respectively and the reduction lasted for more than 15 days. In contrast, blood pressure was continuously increased in the vehicle controls as well as vector controls. The ratio of heart weight to and left ventricular weight to body weight were significantly lower in ACE-RNAi rats, AT1RRNAi rats and ACE-AT1R-RNAi rats respectively than in the vehicle $(p<0.05)$ and vector controls $(p<0.05)$. Myocardial pathology and ultrastructure were also significantly improved in ACE-RNAi rats, AT1R-RNAi rats and ACE-AT1R-RNAi rats, compared with that in vehicle and vector controls.

Conclusions ACE and AT1R silencing had significant antihypertensive effects and reversed hypertensive-induced cardiac hypertrophy in SHR, so RNAi might be a new strategy in control hypertension.

\section{e0158 TRANSIENT PREHYPERTENSIVE TREATMENT IN SPONTANEOUSLY HYPERTENSIVE RATS: A COMPARISON OF LOSARTAN AND AMLODIPINE REGARDING LONG-TERM BLOOD PRESSURE AND CARDIAC PROTECTION}

doi:10.1136/hrt.2010.208967.158

Jinxiu Lin, Liming Lin, Hong Tang, Changsheng Xu. Department of Cardiology, The First Affiliated Hospital of Fujian Medical University, Fujian Hypertension Research Institute, Fuzhou, China

Aims To compare the effectiveness of transient prehypertensive treatment with losartan versus amlodipine in spontaneously hypertensive rats (SHR) on long-term blood pressure and cardiac protection Main methods SHR were prehypertensively (weeks 4-10 of age) treated with losartan (SHR-Los: $20 \mathrm{mg} / \mathrm{kg} /$ day), amlodipine (SHR-Aml: $10 \mathrm{mg} / \mathrm{kg} /$ day) or saline ( $\mathrm{n}=24$ each group). Rats were followed up until week 46. Systolic blood pressure (SBP) was measured by tail-cuff method. Cardiac parameters including Left ventricular (LV) mass index (LVMI), collagen volume fraction (CVF) and LV function were assessed by histomorphometry and echocardiography. Plasma and myocardium angiotensin II (Ang II) and aldosterone (Aldo) were measured by radioimmunoassay. Cardiac angiotensin II type 1 and type 2 receptor (AT1R and AT2R) protein were determined by immunoblotting and brain natriuretic peptide (BNP) mRNA was semi-quantified by reverse transcription-PCR (RT-PCR).

Key findings The SBP in SHR-Los was reduced until age 46 weeks, but returned to untreated SHR levels in SHR-Aml from 30 weeks onwards. Compared to untreated SHR, the LVMI and CVF in SHRLos were markedly decreased until week 46, and the LV ejection fraction (LVEF) (SHR-Los vs SHR: $83.1 \pm 2.3 \%$ vs $79.5 \pm 1.9 \%$, $\mathrm{p}<0.05)$ and cardiac BNP mRNA expression were improved, whereas comparable LVMI and elevated CVF were found in SHRAml, and the LVEF fell significantly below that of untreated SHR at week 46 (SHR-Aml vs SHR: $74.4 \pm 4.3 \%$ vs $79.5 \pm 1.9 \%, p<0.05$ ), with cardiac BNP mRNA expression increasing slightly. Compared to untreated SHR, the plasma and myocardium Ang II and Aldo levels in SHR-Los at week 46 were remarkably decreased (plasma Ang II: $302 \pm 32$ vs $458 \pm 32 \mathrm{pg} / \mathrm{ml}$; plasma Aldo: $172 \pm 20$ vs $252 \pm 41 \mathrm{pg} / \mathrm{ml}$; cardiac Ang II: $126 \pm 11$ vs $199 \pm 14 \mathrm{pg} / 100 \mathrm{mg}$; cardiac Aldo: $497 \pm 43$ vs $766 \pm 46 \mathrm{pg} / 100 \mathrm{mg}$, all $\mathrm{p}<0.05$ ), and the cardiac AT1R protein was down-regulated and AT2R protein was up-regulated, no significant difference of these indices was found between SHR-Aml and untreated SHR. Significance Prehypertensive treatment with losartan was more effective than amlodipine on delaying long-term blood pressure rise and meliorating cardiac structure and function, which might be related to permanent attenuation of circulating and local renin-angiotensin (R-A) systems.

\section{e0159 OSTEOPONTIN IS INVOLVED IN UROTENSIN II INDUCED MIGRATION OF ADVENTITIAL FIBROBLASTS FROM RAT AORTA}

doi:10.1136/hrt.2010.208967.159

${ }^{1}$ Zhang Yonggang, ${ }^{1}$ Kuang Zejian, ${ }^{1}$ Mao Yanyan, ${ }^{2}$ Wei Ruihong, ${ }^{1}$ Bao Shilin, ${ }^{1}$ Wu Libiao, ${ }^{1} \mathrm{Hu}$ Yanchao. ${ }^{1}$ First Affiliated Hospital, Shantou University Medical College, Shantou, China; ${ }^{2}$ Second Affiliated Hospital, Shantou University Medical College, Shantou, China

Background Recent studies suggest osteopontin (OPN) plays a critical role in the progression of vascular remodelling, and that Urotensin II (UII) is a potent vasoconstrictor and stimulator of cellular migration. The goal of this study was to test the hypothesis that OPN is involved in UII-induced migration of rat aortic adventitial fibroblasts (AFs), and examine the effect and mechanisms of UII on OPN expression. 